\title{
Numerical treatment of retarded radiation effects from high brightness electron beams
}

\author{
A. R. Rossi, A. Bacci, and L. Serafini \\ INFN-MI, via Celoria 16, I-20133 Milano, Italy \\ C. Maroli and V. Petrillo \\ Dipartimento di Fisica, Università degli Studi di Milano, via Celoria 16, I-20133 Milano, Italy
}

(Received 21 April 2009; published 20 October 2009)

\begin{abstract}
A form is given of the retarded electric field and magnetic induction generated by the motion of a charged particle that expresses these fields as integrals of the retarded charge density only, with kernels depending on the charge velocity and acceleration fields. In the case of a single pointlike charge, the usual Liénard-Wiechert fields follow very easily. The set of equations for the dynamics of particles in assigned electromagnetic fields with the self-consistent field is written and integrated. The code RETAR for the dynamics of charged particles in external and self-consistent fields is described and a few examples of benchmark are proposed. As a physical application, the case of an electron beam moving in a bending magnetic dipole is examined, and the radiation produced analyzed, in order to characterize a therahertz radiation source.
\end{abstract}

DOI: $10.1103 /$ PhysRevSTAB.12.104202

PACS numbers: 41.60. $-\mathrm{m}, 41.75 .-\mathrm{i}, 41.20 .-\mathrm{q}$

\section{INTRODUCTION}

In this paper we will analyze the radiation emitted by charged bodies moving in assigned external electric and magnetic forces, taking into account the effect of selfconsistent fields. In particular, we will derive the general form of the equations used for describing the dynamics of a beam of charged particles (in our case, electrons) in relativistic motion under the action of assigned electromagnetic fields and under the self-consistent forces. The problem is well known and has been solved, both analytically and numerically, in numerous applications for what concerns the effects of external electric and magnetic fields [1]. As regards the present problem of the focusing and acceleration of electron bunches in the electromagnetic structures of photoinjectors and in linacs, there exist many codes that give the whole start-to-end beam dynamics in a wide set of experimental situations [2-9]. Apart from the code TREDI [6,7], the contribution of selfconsistent terms to the total field is usually calculated using various degrees of approximation, from a quasistatic limit to the use of Green functions. We are interested in the problem of calculating the contribution of self-consistent and radiation fields in the most general setting. We will therefore show how to write the self-consistent force in a way particularly suitable for numerical calculations and present the code RETAR $[10,11]$ together with its application in cases where the self-consistent fields are important and the radiation emitted is intense. The code has been tested in a few simple single particle examples, and then applied in more complex and realistic situations.

The plan of the paper is the following: in Sec. II we will show the form of the retarded field that we will use in our code and that is particularly suitable for numerical application. The architecture of the code RETAR is described in Sec. III, while in Sec. IV few single particle benchmarking examples, like the calculation of the comoving field of one electron, the radiation due to the motion inside a uniform electric field, and the synchrotron radiation, are presented. In Sec. V we show the results of two multiparticle simulations, namely, the extraction of an electron bunch from a photocathode and the evaluation of radiation when a beam moves inside a bending magnetic dipole focusing on the application of this system as a terahertz source or a diagnostic device. Finally we present some conclusions.

\section{THE MODEL EQUATIONS}

The electromagnetic fields generated by a charged body in motion are given by the well-known "retarded" solution of Maxwell's equations in vacuum [i.e., the charge density $\rho(\mathbf{x}, t)$ and current density $\mathbf{J}(\mathbf{x}, t)$ are the only sources appearing in the equations] [1].

In general terms, the retarded solution of the nonhomogeneous partial differential equation

$$
\left(\frac{\partial^{2}}{\partial t^{2}}-c^{2} \nabla^{2}\right) f(\mathbf{x}, t)=S(\mathbf{x}, t)
$$

is identified with the following particular solution:

$$
f_{\text {ret }}(\mathbf{x}, t)=\frac{1}{4 \pi c^{2}} \int d \mathbf{x}^{\prime} \frac{S_{\text {ret }}}{\left|\mathbf{x}-\mathbf{x}^{\prime}\right|},
$$

where $S_{\text {ret }}=S\left(\mathbf{x}^{\prime}, t_{\text {ret }}\right)$ and $t_{\text {ret }}=t-\frac{1}{c}\left|\mathbf{x}-\mathbf{x}^{\prime}\right|$.

One usually starts from the electromagnetic scalar and vector potentials $\Phi(\mathbf{x}, t)$ and $\mathbf{A}(\mathbf{x}, t)$ in the Lorentz gauge, 
because the nonhomogeneous terms $S_{\Phi}(\mathbf{x}, t)$ and $\mathbf{S}_{\mathrm{A}}(\mathbf{x}, t)$ in the corresponding equations of the form (1) are particularly simple, namely $S_{\Phi}(\mathbf{x}, t)=4 \pi c^{2} \rho(\mathbf{x}, t)$ and $\mathbf{S}_{\mathrm{A}}(\mathbf{x}, t)=4 \pi c \mathbf{J}(\mathbf{x}, t)$. In this way, one obtains (in Gaussian units), the well-known retarded forms of the electromagnetic potentials, i.e.,

$$
\Phi_{\text {ret }}(\mathbf{x}, t)=\int d \mathbf{x}^{\prime} \frac{\rho_{\text {ret }}}{\left|\mathbf{x}-\mathbf{x}^{\prime}\right|}
$$

and

$$
\mathrm{A}_{\text {ret }}(\mathbf{x}, t)=\frac{1}{c} \int d \mathbf{x}^{\prime} \frac{\mathbf{J}_{\mathrm{ret}}}{\left|\mathbf{x}-\mathbf{x}^{\prime}\right|} .
$$

The even more common Liénard-Wiechert form of the retarded potentials [1] follows when $\mathbf{E}_{\text {ret }}$ and $\mathbf{B}_{\text {ret }}$ are needed only at large distances from the sources $\rho(\mathbf{x}, t)$ and $\mathbf{J}(\mathbf{x}, t)$ that are supposed to be, in this case at least, well localized functions in space at all times.

It is also possible to start from equations of the form (1), directly for the electric field $\mathbf{E}$ and magnetic induction $\mathbf{B}$, in which case the source terms $\mathbf{S}_{\mathrm{E}}$ and $\mathbf{S}_{\mathrm{B}}$ have the somewhat more complex expressions

$$
\begin{aligned}
& \mathbf{S}_{\mathrm{E}}=-4 \pi\left(\frac{\partial}{\partial t} \mathbf{J}+c^{2} \nabla \rho\right) \\
& \mathbf{S}_{\mathrm{B}}=4 \pi c \nabla \times \mathbf{J} .
\end{aligned}
$$

In the corresponding integrals that give the retarded solutions $\mathbf{E}_{\text {ret }}$ and $\mathbf{B}_{\text {ret }}$, we will then perform suitable integrations by parts in order to present the fields in more concise and, in the end, simpler expressions. We think that the final forms of the electromagnetic fields that may be reached in this way and that are explicitly given in Eqs. (12) and (13) may also be of help in numerical works, when (i) one wants to calculate the fields generated by any given motion of the system [i.e., by any given pair of functions $\rho(\mathbf{x}, t)$ and $\mathbf{J}(\mathbf{x}, t)]$ and also (ii) when it is necessary or more simply desirable to take account of the reactions of the fields on the motion of the particles.

First of all, and without loss of generality, it is convenient to represent the current density by introducing explicitly the velocity field of the charges defined as $\mathbf{v}(\mathbf{x}, t)=\mathbf{J}(\mathbf{x}, t) / \rho(\mathbf{x}, t)$, namely, by writing the current density in the form $\mathbf{J}(\mathbf{x}, t)=\rho(\mathbf{x}, t) \mathbf{v}(\mathbf{x}, t)$. The two source terms (5) can then be written as

$$
\begin{aligned}
& \mathbf{S}_{\mathrm{E}}=-4 \pi\left[\left(\mathbf{a}-c^{2} \beta \nabla \cdot \beta\right) \rho+c^{2}(\overleftrightarrow{\mathbf{1}}-\beta \beta) \cdot \nabla \rho\right] \\
& \mathbf{S}_{\mathrm{B}}=4 \pi c^{2}[\rho \nabla \times \beta-\beta \times \nabla \rho]
\end{aligned}
$$

where $\beta(\mathbf{x}, t)=\frac{1}{c} \mathbf{v}(\mathbf{x}, t), \stackrel{\leftrightarrow}{\mathbf{1}}$ is the unit dyadic, and we have also introduced the acceleration field $\mathbf{a}(\mathbf{x}, t)=\partial_{t} \mathbf{v}(\mathbf{x}, t)$.

As we will apply this model to a beam of particles, we shall assume $\rho=\sum_{j} \rho_{j}$, where the set of $\rho_{j}$ is a generic space discretization of $\rho$ and, for each $\rho_{j}$, that both velocity and acceleration fields actually depend only on retarded time, i.e., that $\mathbf{v}_{j}=\mathbf{v}_{j}\left(t_{\text {ret }}\right)$ and $\mathbf{a}_{j}=\mathbf{a}_{j}\left(t_{\text {ret }}\right)=\left.\frac{d}{d t} \mathbf{v}_{j}(t)\right|_{t=t_{\text {ret }}}$. The retarded electric field generated by $\rho_{j}$ can be written as $\mathbf{E}_{\text {ret }}^{(j)}=\mathbf{E}_{1}^{(j)}+\mathbf{E}_{2}^{(j)}$, with the two partial vectors $\mathbf{E}_{1}^{(j)}$ and $\mathbf{E}_{2}^{(j)}$ given by

$$
\begin{aligned}
& \mathbf{E}_{1}^{(j)}=-\frac{1}{c^{2}} \int \frac{d \mathbf{x}^{\prime}}{\left|\mathbf{x}-\mathbf{x}^{\prime}\right|} \mathbf{a}_{j}\left(t_{\mathrm{ret}}\right) \rho_{\mathrm{ret}}^{(j)} \\
& \mathbf{E}_{2}^{(j)}=-\int \frac{d \mathbf{x}^{\prime}}{\left|\mathbf{x}-\mathbf{x}^{\prime}\right|}\left[\stackrel{\leftrightarrow}{\mathbf{1}}-\beta_{j}\left(t_{\mathrm{ret}}\right) \beta_{j}\left(t_{\mathrm{ret}}\right)\right] \cdot\left(\nabla \rho^{(j)}\right)_{\mathrm{ret}} .
\end{aligned}
$$

From now on, for the sake of simplicity, we will drop the index $j$; the total field can be calculated, by superposition principle, summing over all contributions. The vector $(\nabla \rho)_{\text {ret }}$ under the integral sign is connected to the vector $\nabla^{\prime}(\rho)_{\text {ret }}=\nabla^{\prime} \rho\left(\mathbf{x}^{\prime}, t-\frac{1}{c}\left|\mathbf{x}-\mathbf{x}^{\prime}\right|\right)$ (where $\nabla^{\prime}$ means the gradient with respect to the primed spatial variable $\mathbf{x}^{\prime}$ ), through the chain

$$
\begin{aligned}
\nabla^{\prime}(\rho)_{\mathrm{ret}} & =(\nabla \rho)_{\mathrm{ret}}+\left(\partial_{t} \rho\right)_{\mathrm{ret}}\left(\nabla^{\prime} t\right)_{\mathrm{ret}} \\
& =(\nabla \rho)_{\mathrm{ret}}-\left(\nabla^{\prime} t\right)_{\mathrm{ret}} \mathbf{v}\left(t_{\mathrm{ret}}\right) \cdot(\nabla \rho)_{\mathrm{ret}} \\
& =(\nabla \rho)_{\mathrm{ret}} \cdot\left[\stackrel{\leftrightarrow}{\mathbf{1}}-\mathbf{v}\left(t_{\mathrm{ret}}\right)\left(\nabla^{\prime} t\right)_{\mathrm{ret}}\right]
\end{aligned}
$$

where use has been made of the charge conservation.

Also, noting that $\nabla^{\prime} t_{\text {ret }}=\nabla^{\prime}\left(t-\frac{1}{c}\left|\mathbf{x}-\mathbf{x}^{\prime}\right|\right)=\frac{1}{c} \mathbf{n}$, where the unit vector $\mathbf{n}=\frac{\mathbf{x}-\mathbf{x}^{\prime}}{\left|\mathbf{x}-\mathbf{x}^{\prime}\right|}$, we have

$$
(\nabla \rho)_{\mathrm{ret}}=\nabla^{\prime}(\rho)_{\mathrm{ret}} \cdot \stackrel{\leftrightarrow}{\mathbf{T}}
$$

with the $3 \times 3$ dyadic $\stackrel{\leftrightarrow}{\mathbf{T}}$ given by $\stackrel{\leftrightarrow}{\mathbf{T}}=\stackrel{\leftrightarrow}{\mathbf{1}}+\frac{\beta \mathbf{n}}{1-\mathbf{n} \cdot \beta}$. By inserting (9) in (7b) one is able to do an integration by parts, which reduces the partial field $\mathbf{E}_{2}$ to the form

$$
\mathbf{E}_{2}=\int d \mathbf{x}^{\prime}\left[\nabla^{\prime} \cdot\left(\frac{\stackrel{\leftrightarrow}{\mathbf{Q}}}{\left|\mathbf{x}-\mathbf{x}^{\prime}\right|}\right)\right] \rho_{\text {ret }}
$$

where the new dyadic $\overleftrightarrow{\mathbf{Q}}$ is $\overleftrightarrow{\mathbf{Q}}=\overleftrightarrow{\mathbf{1}}+\frac{\beta(\mathbf{n}-\beta)}{1-\mathbf{n} \cdot \beta}$. In conclusion, we may write the following expression for the retarded electric field:

$$
\mathbf{E}_{\mathrm{ret}}(\mathbf{x}, t)=\int d \mathbf{x}\left[\nabla^{\prime} \cdot\left(\frac{\stackrel{\leftrightarrow}{\mathbf{Q}}}{\left|\mathbf{x}-\mathbf{x}^{\prime}\right|}\right)-\frac{1}{c^{2}} \frac{\mathbf{a}\left(t_{\mathrm{ret}}\right)}{\left|\mathbf{x}-\mathbf{x}^{\prime}\right|}\right] \rho_{\mathrm{ret}} .
$$

To calculate the primed divergence of the dyadic $\frac{\overleftrightarrow{\mathbf{Q}}}{\left|\mathbf{x}-\mathbf{x}^{\prime}\right|}$ appearing under the integral sign in the preceding equation is lengthy but straightforward, with the final result

$$
\begin{aligned}
\mathbf{E}_{\mathrm{ret}}(\mathbf{x}, t)= & \int d \mathbf{x}^{\prime}\left\{\frac{\mathbf{n} \times[(\mathbf{n}-\beta) \times \dot{\beta}]}{c\left|\mathbf{x}-\mathbf{x}^{\prime}\right|(1-\mathbf{n} \cdot \beta)^{2}}\right. \\
& \left.+\frac{\mathbf{n}-\beta}{\gamma^{2}\left|\mathbf{x}-\mathbf{x}^{\prime}\right|^{2}(1-\mathbf{n} \cdot \beta)^{2}}\right\} \rho_{\mathrm{ret}},
\end{aligned}
$$


where $\dot{\beta}(t)=\frac{d}{d t} \beta(t)$ and $\gamma^{2}(t)=\left[1-\beta^{2}(t)\right]^{-1}$ must be calculated at $t=t_{\text {ret }}$ and $\mathbf{n}$ as defined before.

Working out the same kind of calculations for the magnetic induction leads to the companion expression

$$
\begin{aligned}
\mathbf{B}_{\text {ret }}(\mathbf{x}, t)= & \int d \mathbf{x}^{\prime}\left\{\frac{\mathbf{n} \times[\dot{\beta}(1-\mathbf{n} \cdot \beta)+\beta(\dot{\beta} \cdot \mathbf{n})]}{c\left|\mathbf{x}-\mathbf{x}^{\prime}\right|(1-\mathbf{n} \cdot \beta)^{2}}\right. \\
& \left.+\frac{\mathbf{n} \times \beta}{\gamma^{2}\left|\mathbf{x}-\mathbf{x}^{\prime}\right|^{2}(1-\mathbf{n} \cdot \beta)^{2}}\right\} \rho_{\text {ret }}
\end{aligned}
$$

These same forms for $\mathbf{E}_{\text {ret }}$ and $\mathbf{B}_{\text {ret }}$ could also be obtained, of course, by starting directly from the retarded potentials in Eqs. (3) and (4), following the general rule $\mathbf{E}=$ $-\frac{1}{c} \partial_{t} \mathbf{A}-\nabla \Phi, \mathbf{B}=\nabla \times \mathbf{A}$, and again using Eq. (8).

In the particular case in which $\rho(\mathbf{x}, t)=q \delta[\mathbf{x}-\mathbf{r}(t)]$, i.e., when the retarded fields are considered to be produced by a single pointlike source of charge $q$ with instantaneous position and velocity $\mathbf{r}(t)$ and $\mathbf{v}(t)=\frac{d}{d t} \mathbf{r}(t)$, Eqs. (12) and (13) can be quickly rewritten in the usual Liénard-Wiechert form, the extra form factor $(1-\mathbf{n} \cdot \beta)$ in the denominators coming from the known properties of the delta function.

If we are interested in the relativistic dynamics of a beam of charged particles in assigned external fields $\mathbf{E}_{\text {est }}$ and $\mathbf{B}_{\text {est }}$, the equations of motion write as

$$
\begin{aligned}
\frac{d \mathbf{x}_{j}}{d t} & =\mathbf{v}_{j} \\
\frac{d \mathbf{p}_{j}}{d t} & =q_{j}\left(\mathbf{E}_{\mathrm{tot}}+\beta_{j} \times \mathbf{B}_{\mathrm{tot}}\right),
\end{aligned}
$$

where the index $j$ runs over the total number of charges $q_{j}$, representing a particular implementation of the charge density discretization, $\mathbf{E}=\mathbf{E}_{\text {est }}+\mathbf{E}_{\text {ret }}$ and $\mathbf{B}=$ $\mathbf{B}_{\text {est }}+\mathbf{B}_{\text {ret }}$ are the total fields, and $\mathbf{p}_{j}=m_{j} \gamma_{j} \mathbf{v}_{j}$.

\section{THE ARCHITECTURE OF THE NUMERICAL CODE RETAR}

In the following we will describe the code RETAR for particle and beam dynamics. First of all, the electrons of the beam are represented by macroparticles, whose charge is $q=-e N_{\mathrm{mp}}$, with $N_{\mathrm{mp}}$ number of electrons over each one. The integration of the relativistic equations of motion for the macroparticles is performed with a fifth order Runge-Kutta method. The coordinates of all macroparticles, together with their velocities and accelerations, are stored for all times and used to evaluate the self-consistent forces on the particles themselves, as presented in Eq. (14). Coordinates, velocities, and accelerations are also used to compute the radiation fields in the points of the space, where they are required. The code RETAR can compute the retarded fields in two different modes, depending on the electrons motion. If the run is short and the particles move under the effect of external fields in cases where the emission can be relevant, an appropriately small constant time step $d t$ is used, and the self-consistent fields are computed directly by means of (12) and (13), dividing the space in spherical shells around the center of mass of the particle; such shells hold particles with the same (average) retarded time, which is equal, setting $c=1$, to the shell's average radius. This procedure prevents a direct calculation of the retarded time, providing it with a maximum error in its determination given by the shell half thickness (i.e. $d t / 2$ ). Each shell is then divided in small cells, with typical linear dimension $c d t$ of tens of microns, and the mean velocity and acceleration of the electrons that were inside the cells at the retarded time $t_{\text {ret }}$ are evaluated.

If long drifts are present in the beam line and a procedure of variable time steps is required, the code RETAR uses the usual Liénard-Wiechert expression of the fields for point particles, evaluating the retarded time numerically on the base of the past dynamics by means of a Newton-Raphson scheme.

The presence of point macroparticles can introduce numerical problems due to the nonphysically high charges associated. We then model our particles as small spheres (see, for example [6]) whose radius is chosen such that their total volume is equal to the beam rms volume. Nevertheless a number of macroparticles in excess of 5000 is usually needed to prevent unphysical particles scattering.

When the particles are extracted by photocathodes, as in the application to high brightness electron beams, a particular procedure of charge extraction is activated (see Sec. VA).

\section{Parallelization}

RETAR has been parallelized with the message passing interface library and a sketch of its logical flow is shown in Fig. 1. The cluster is divided in two communicators: node 0 is isolated from the others since its task consists in calculating collective average parameters (such as beam size, emittance, ...) and saving on the hard disk phase spaces and any other data produced. Moreover, it places the macroparticles close to the cathode if the simulation involves an $\mathrm{rf}$ gun. Nodes from 1 to $m$ actually perform the simulation. The whole set of $\mathrm{P}=\left\{p_{1}, p_{2}, \ldots\right.$, $\left.p_{m}\right\}$ macroparticles is equally divided between the remaining $m$ nodes, as is the whole history matrix $\mathcal{H}_{\mathrm{P}}$, which keeps track of the past kinematics, so that each node only manages its subset of particles $p_{i}$, together with their history $\mathcal{H}_{p_{i}}$. This allows one to distribute memory usage evenly on the cluster and balance the computational load.

At the beginning of each time step, every node $i$ has in memory the whole bunch phase space $\mathcal{C}_{\mathrm{P}}(t)$ computed at the previous step. Then it calculates the contribution to the self-fields $\mathcal{F}_{\mathrm{P}}^{i}$, acting on each particle, due to its particle subset $p_{i}$ and shares the result with the other nodes, where it is summed up to obtain the whole internal field $\mathcal{F}_{\mathrm{P}}$ acting on each node's particles. At the end of this stage, each node is able to apply the Runge-Kutta $(\mathcal{R} \mathcal{K})$ to his subset of 


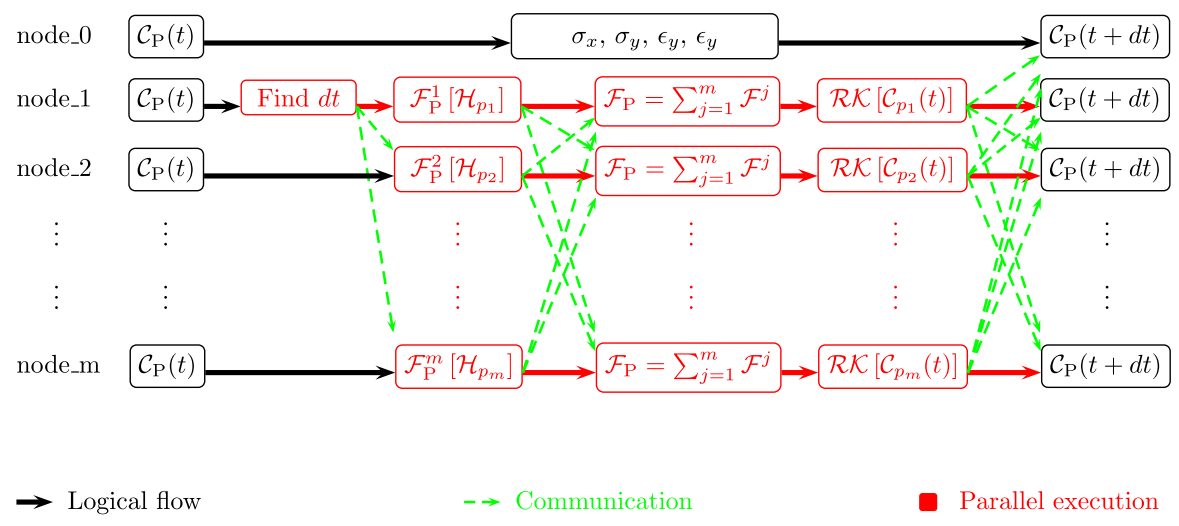

FIG. 1. (Color) Sketch of RETAR logical flow.

particles $p_{i}$ and share the result with the others; at the end of the time step, each node has in memory $\mathcal{C}_{\mathrm{P}}(t+d t)$. Since setting the step size can be very time costly, due to failures in obtaining the required accuracy, it is only performed on the subset $p_{1}$ and the result communicated to the other nodes.

\section{CODE BENCHMARKING}

In previous works [10-12], we have compared the average characteristics of a beam in a beam line, such as the envelope and the emittance, with those given by other codes, obtaining a good agreement. We are now interested in testing the capability of RETAR as regards the evaluation of the radiation produced. We will give, therefore, some academic examples useful for benchmarking the code, as the field produced by a charge in rectilinear uniform motion and the relativistic radiation of a single particle in a constant electric field and in a constant magnetic field.

\section{A. The comoving field}

As a first benchmark, we have performed a numerical evaluation of the electric field generated in a point by a particle moving with a constant velocity $\mathbf{v}$ along the $x$ axis. In Fig. 2 the analytical expression of $|\mathbf{E}|$,

$$
|\mathbf{E}|=e \gamma \sqrt{\frac{(x-v t)^{2}+y^{2}}{\left[\left(\gamma^{2}(x-v t)^{2}+y^{2}\right)\right]^{3}}},
$$

is compared versus the numerical values obtained by RETAR in a point $P=(1 \mathrm{~mm}, 0.2 \mathrm{~mm}, 0)$ for $p_{0} / m_{0} c=$ 0.2 . The difference observed for times less than $4 \times$ $10^{-14} \mathrm{~s}$ depends on the fact that the analytical formulas are obtained under the hypothesis of a uniform motion starting at $t=-\infty$, while the code begins the motion at $t=0$, so the comoving field reaches $P$ after a time $t=$ $|\mathbf{r}| / c$.

\section{B. Radiation from a particle in a constant electric field}

The second example concerns the radiation emitted by a particle in a static electric field. The external electric field considered is along the $z$ axis and is

$$
\mathbf{E}(z)= \begin{cases}E_{0} \mathbf{e}_{z} & \text { for } 0<z<z_{\text {field }} \\ 0 & \text { for } z<0 \text { and } z>z_{\text {field }}\end{cases}
$$

The equations of motion inside the field, supposing $x_{0}=$ $y_{0}=0$ and $\mathbf{p}_{0}=p_{0} \mathbf{e}_{z}$, can be solved analytically, and give

$$
\begin{aligned}
z(t) & =\frac{c\left(\sqrt{m_{0}^{2} c^{2}+\left(p_{0}+e E_{0} t\right)^{2}}-P_{0}\right)}{e E_{0}}+z_{0} \\
\beta(t) & =\frac{p_{0}+e E_{0} t}{\sqrt{m_{0}^{2} c^{2}+\left(p_{0}+e E_{0} t\right)^{2}}},
\end{aligned}
$$

where $P_{0}=\sqrt{m_{0}^{2} c^{2}+p_{0}^{2}}$. The retarded time generating

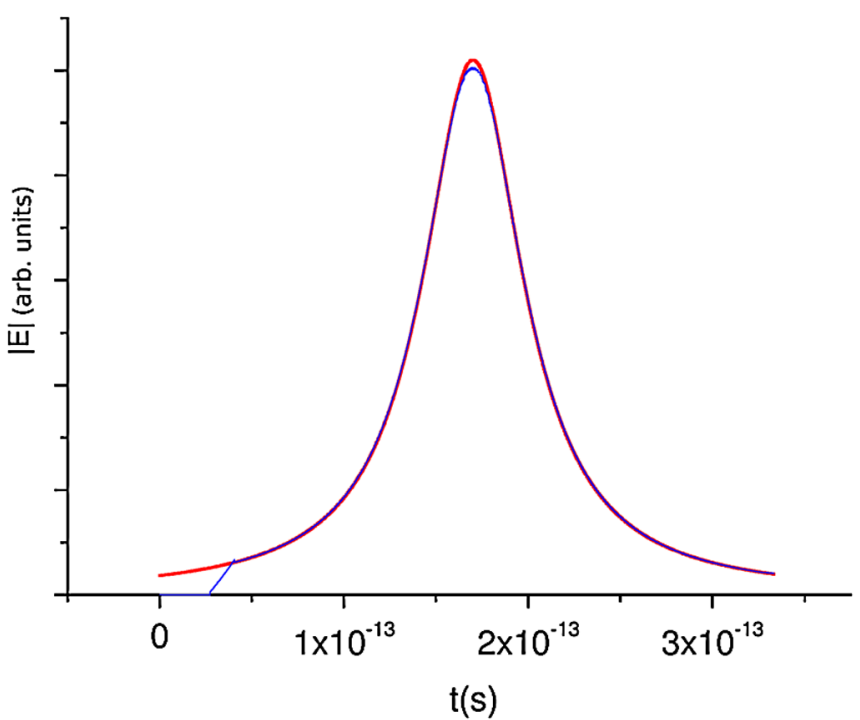

FIG. 2. (Color) The comoving field calculated by RETAR (blue) compared to its analytic value (red). 
the signal at the points $P_{\mathrm{s}}=\left(x_{\mathrm{s}}, y_{\mathrm{s}}, z_{\mathrm{s}}\right)$ on a flat screen in the $(x, y)$ plane can be computed and turns out to be

$$
\begin{aligned}
t_{\text {ret }}= & \frac{t}{2}-\frac{p_{0}}{2 e E_{0}}+\frac{1}{2\left[c^{2}\left(p_{0}+e E_{0} t\right)^{2}-\left(c P_{0}+e E_{0} z_{\mathrm{s}}\right)^{2}\right]} \\
& \times\left\{( P _ { 0 } + \frac { e E _ { 0 } z _ { \mathrm { s } } } { c } ) \left[4 c^{4} m_{0}^{2} r^{2}+\left(e E_{0}\left(R^{2}-c^{2} t^{2}\right)\right.\right.\right. \\
& \left.\left.+2 c\left(P_{0} z_{\mathrm{s}}-c p_{0} t\right)\right)^{2}\right]^{1 / 2} \\
& \left.-\left(\frac{p_{0}}{e E_{0}}+t\right)\left(c^{4} m_{0}^{2}+e^{2} E_{0}^{2} r^{2}\right)\right\},
\end{aligned}
$$

where $r^{2}=\sqrt{x_{\mathrm{s}}^{2}+y_{\mathrm{s}}^{2}}$ and $R^{2}=\sqrt{r^{2}+z_{\mathrm{s}}^{2}}$. On the basis of this expression of the retarded time, we can calculate analytically the expression of the radiation terms of the fields, and then, integrate numerically the flux of the Poynting vector through the surface $\Sigma$ representing the screen, obtaining the total power collected:

$$
P=\frac{c}{4 \pi} \int_{\Sigma} d \Sigma(\mathbf{E} \times \mathbf{B}) \cdot \mathbf{m}
$$

where $\mathbf{m}$ is the unit vector normal to $d \mathbf{\Sigma}$. Assuming an electron moving along the $z$ direction, and $z_{\text {field }}=1 \mathrm{~cm}$, $z_{\mathrm{s}}=2 \mathrm{~cm}, E_{0}=5000 \mathrm{StatV} / \mathrm{cm}$ and $p_{0} / m_{0} c=0.16$, the power collected on the screen vs time is given by Fig. 3, where the RETAR results are compared to the theoretical prediction.

\section{Radiation produced in a constant magnetic field}

The third example regards the motion of an electron in a uniform magnetic field, resulting in a circular orbit with frequency $\omega_{\mathrm{c}}=\frac{e B}{\gamma m_{0} c}$. In this case, the comparison has been done between the power emitted by the electron computed by RETAR and the prediction of the Liénard formula:

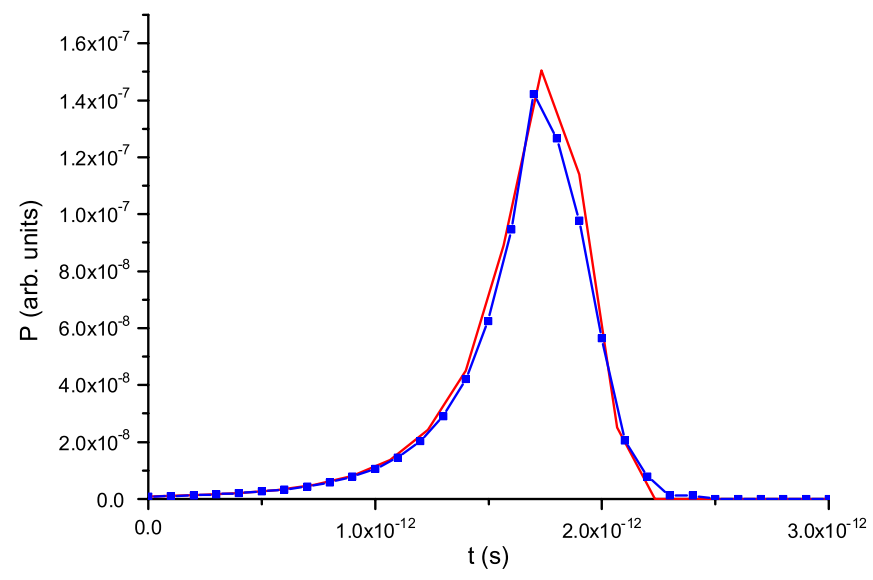

FIG. 3. (Color) The power collected on a flat screen produced by a single charge under the effect of a constant electric field as calculated by RETAR (blue dotted line) compared to the result of Sec. IV B (red line).

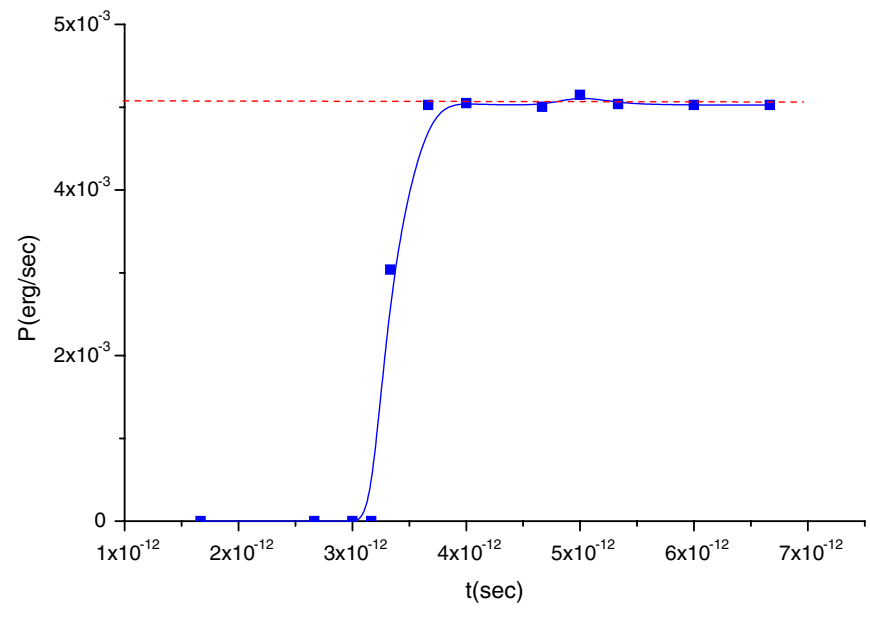

FIG. 4. (Color) The power collected on a sphere produced by a single charge under the effect of a constant magnetic field as calculated by RETAR (blue dotted line) compared to the result of the Liénard formula (21) (red dashed line).

$$
P=\frac{2}{3} \frac{q^{2}}{c} \gamma^{4}\left[|\dot{\beta}|+\gamma^{2}|\beta \cdot \dot{\beta}|\right] .
$$

The power collected in the solid angle turns out to be constant at the value

$$
P=\frac{2}{3} \frac{e^{4} B^{2}}{m_{0}^{2} c^{3}}\left(\gamma^{2}-1\right)
$$

The contributions in a point $\left(x_{\mathrm{s}}, y_{\mathrm{s}}, z_{\mathrm{s}}\right)$ of a spherical screen with radius $R_{\text {screen }}=1 \mathrm{~cm}$, completely enclosing the electron orbits, and centered in the origin, were produced from the retarded times,

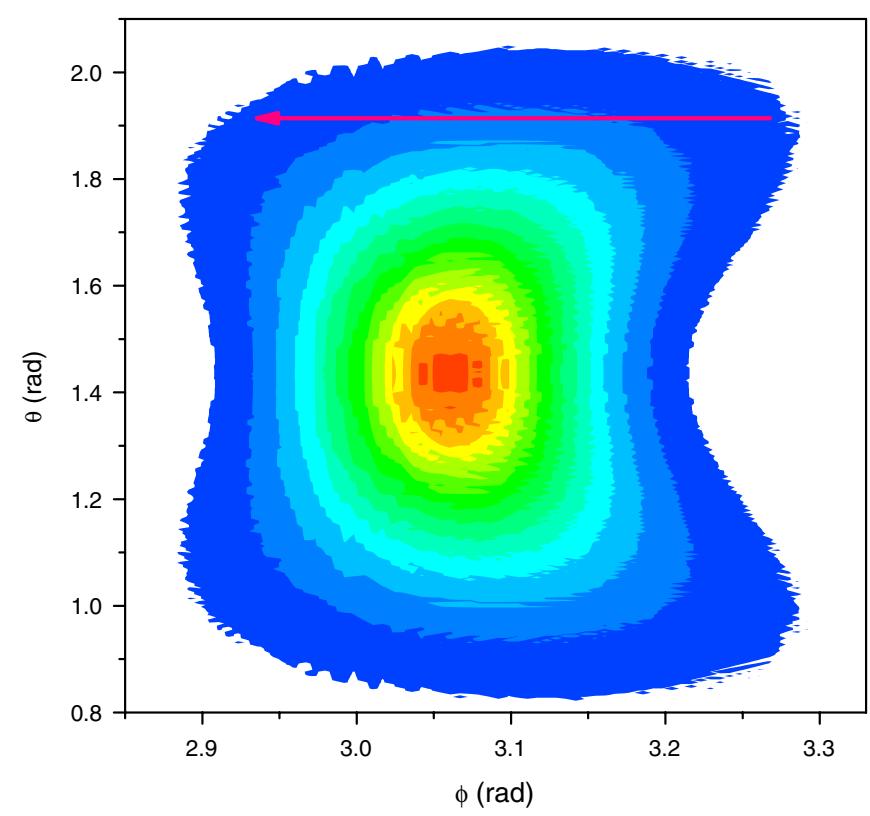

FIG. 5. (Color) The power density collected on a sphere. 


$$
t_{\mathrm{ret}}=t-\sqrt{r_{\mathrm{s}}^{2}-2 \frac{p_{0} c}{e B}\left(x_{\mathrm{s}} \cos \omega_{\mathrm{c}}+z_{\mathrm{s}} \sin \omega_{\mathrm{c}}\right)},
$$

where $r_{\mathrm{s}}^{2}=x_{\mathrm{s}}^{2}+y_{\mathrm{s}}^{2}+z_{\mathrm{s}}^{2}$ and $\mathbf{p}_{0}=p_{0} \mathbf{e}_{z}$. In our case, the magnetic field is $\mathbf{B}=-B \mathbf{e}_{z}$, with $B=10^{5} \mathrm{G}$, the motion is circular in the $(x, y)$ plane, with center in $(0,0)$, and radius $R_{\text {motion }}=683 \mu \mathrm{m}$; the gamma factor is $\gamma=4.123$. In Fig. 4 both theoretical and numerical values are reported, while in Fig. 5 the shape of the radiation versus the spherical coordinates $(\theta, \phi)$ is presented.

The power begins to arrive on the screen at $t=3 \times$ $10^{-12} \mathrm{~s}$ and rapidly grows to the regime value; the shape of the power on the screen shows two bumps forward and the wake in the tail. The arrow shows the direction of the electron motion. The accuracy of the agreement depends upon the integration step on the screen used.

\section{Radiation produced in a bending magnet}

The last single particle benchmark simulates an electron with an energy of $80 \mathrm{MeV}(\gamma=160)$ going through a bending magnet whose field is in the direction of the $x$ axis. The magnet's $z$ profile is a flat plateau $5 \mathrm{~cm}$ long and Gaussian tails $(\sigma=5 \mathrm{~mm})$; the resulting overall longitudinal profile is continuous and smooth so that the coherent synchrotron radiation emission dominates edge radiation. The magnetic field strength is $100 \mathrm{G}$, while the screen, spanning the $(x, y)$ coordinates, measures $10 \times 10 \mathrm{~cm}$, and $z_{\mathrm{s}}=3 \mathrm{~m}$. In Fig. 6 we report the energy density on the screen integrated in time, which agrees remarkably well with the angular distribution predicted by the formula for the distribution of radiation for a charge in instantaneously circular motion with acceleration perpendicular to velocity [1]:

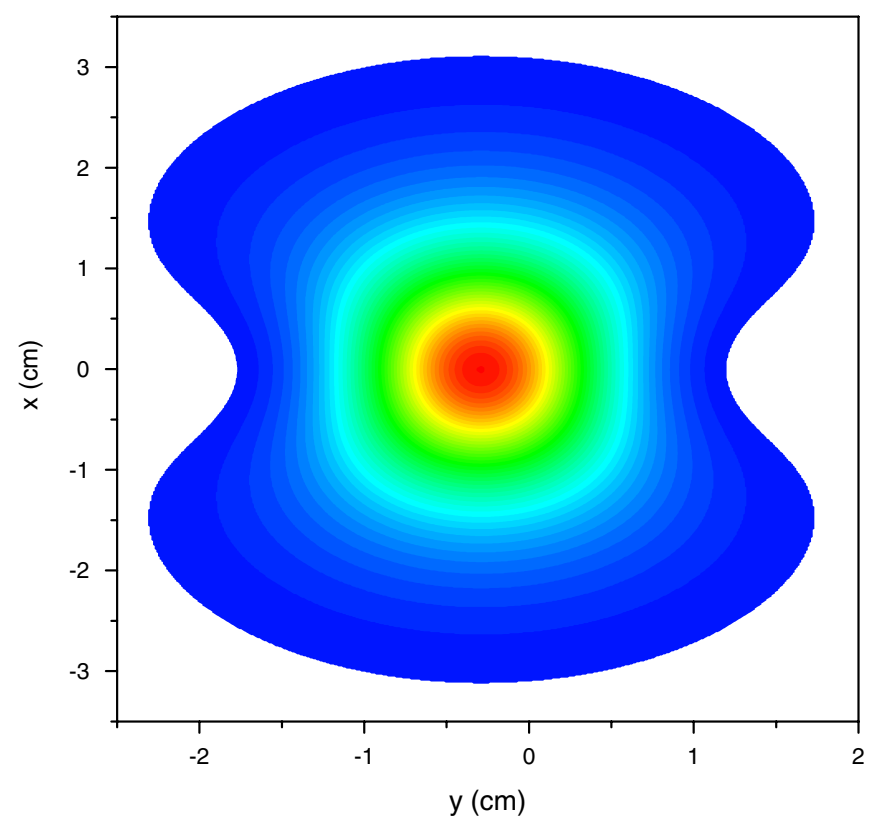

FIG. 6. (Color) The energy density collected on a plane sheet.

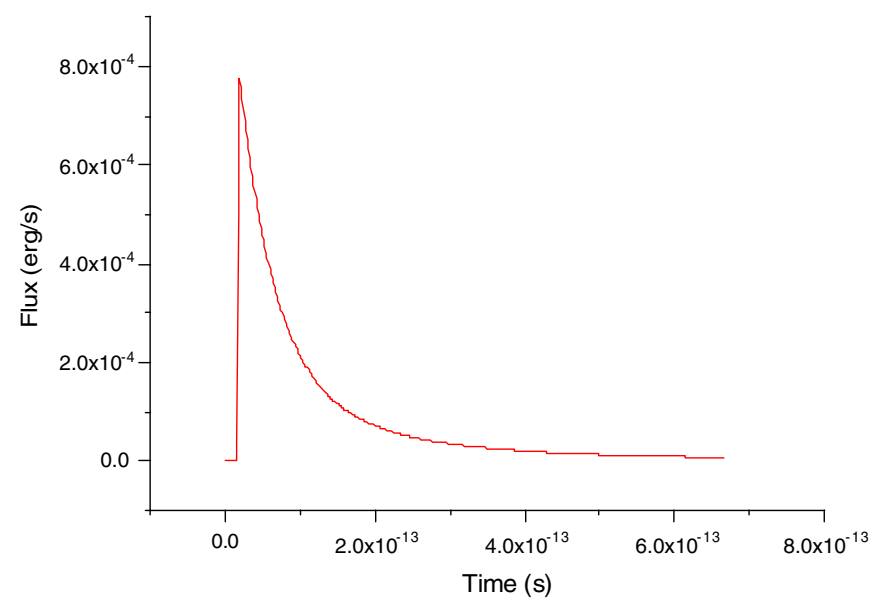

FIG. 7. (Color) Flux of the vector $\mathbf{S}$ as a function of time.

$$
\frac{d P(t)}{d \Omega}=\frac{q^{2}}{4 \pi c^{3}} \frac{|\dot{\mathbf{v}}|^{2}}{(1-\beta \cos \theta)^{3}}\left[1-\frac{\sin ^{2} \theta \cos ^{2} \phi}{\gamma^{2}(1-\beta \cos \theta)^{2}}\right] .
$$

The flux of the Poynting vector through the screen, as a function of time, is shown in Fig. 7: its slowly decaying trend is due to the screen plane shape, while its integral in time represents the total energy crossing the screen itself; it turns out to be $5.56 \times 10^{-17} \mathrm{erg}$, in good agreement with the value of $5.49 \times 10^{-17}$ erg calculated by the Liénard formula with the same kinematics. In order to check whether the angular frequency distribution agrees with theoretical predictions, we collected the $y$ component of the electric field as a function of time for two different $x$ positions [i.e. different $\theta$ values in (23)], namely $x=0$ and $x=2.5 \mathrm{~cm}$, and Fourier transformed them. The results are shown in Fig. 8. Their temporal trend is dominated by the magnet longitudinal profile, since the screen is much larger than the transverse extent of the electron trajectory, seeing the whole trajectory. For this reason, we do not expect the spectrum to show the frequency cutoff,

$$
\omega_{\mathrm{c}}=\frac{3}{2} \gamma^{3}\left(\frac{c}{\rho}\right)
$$

where $\rho$ is the instantaneous radius of curvature; the formula is valid when the radiation collected comes from a very small portion of the trajectory. Nevertheless, as expected, the two results differ in their content of high frequency components, with the $x=0$ spectrum having greater contributions from high frequency, whereas the spectrum taken away from the plane of motion goes to zero much more rapidly. They also differ in the total energy content (as can be deduced by the field intensity) but this effect is hidden by the normalization to unit area of the two spectra.

Finally, we calculated the integrated spectrum averaging the spectra collected at different positions on the screen. The result is shown in Fig. 9. Notice how the spectrum 


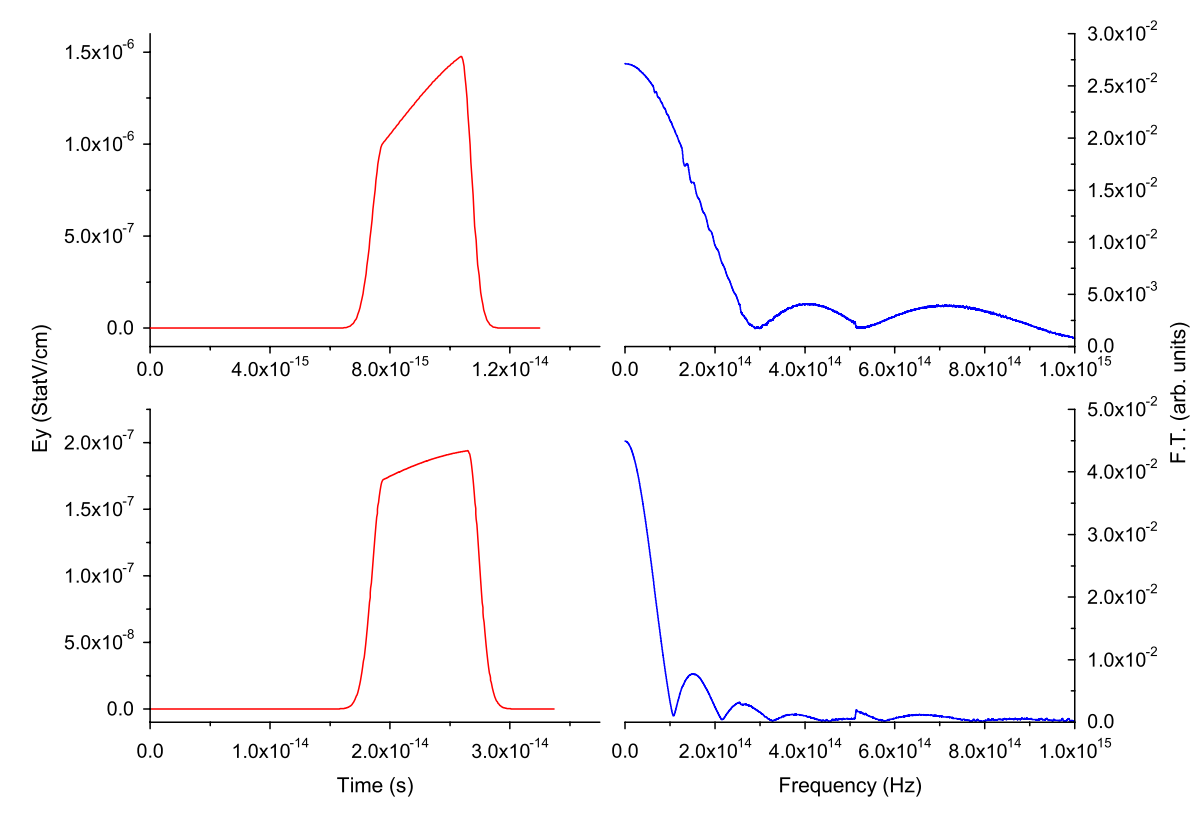

FIG. 8. (Color) Time signals (left) and their Fourier transforms (right) for two different values of $x$.

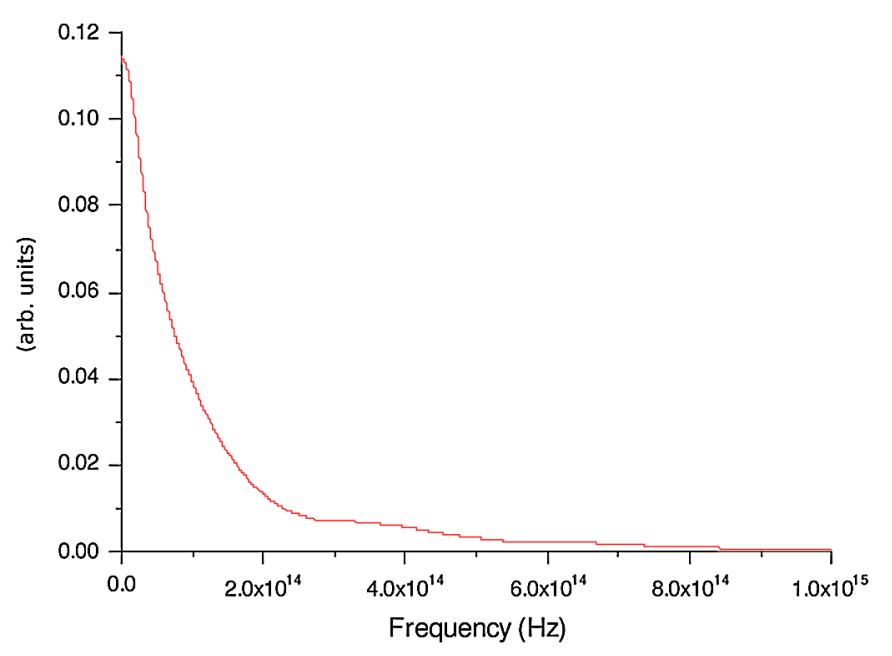

FIG. 9. (Color) Integrated spectrum.

decays exponentially from its maximum up to a frequency of $200 \mathrm{THz}$; the width of the peak is estimated to be $83.1 \pm$ $0.4 \mathrm{THz}$.

\section{APPLICATION TO ELECTRON BUNCHES}

In the following we will describe the application of RETAR to two experimental settings in multiparticle simulations.

\section{A. Bunch extraction from a photocathode}

Modeling the emission of photoelectrons from a photocathode is a delicate task, since the finite extent of temporal steps can introduce numerical stratifications in the longitudinal phase space of the bunch. In order to avoid such a problem, we have modeled the extraction of the charge from the photocathode by means of an algorithm that guarantees the maximum smoothness in the charge distribution without allocating nonphysical values of the initial longitudinal momentum; it connects the structure of the beam generated to the duration of the laser pulse and to the photocathode characteristics. Typical values of the laser pulse duration are about 10 psec for a wavelength $\lambda=$ $0.12 \mu \mathrm{m}$, while the whole time duration of the charge extraction is of about $20-30$ psec, until all the bunches leave the cathode and begin to be driven by the radiofrequency. The cathode itself is considered as a metallic plate set at $z=0$, maintained at null potential by the presence of image charges.

The macroparticles are initially represented by uniformly charged spheres of radius $r_{\mathrm{mp}}$ that, at $t=0$, are placed casually with their centers in a thin volume adjacent to the surface of the cathode, contemporaneously with their images. If a sphere happens to be closer to the cathode than its radius length, its charge (and that of the corresponding image charge) is reduced accordingly to the fraction of volume effectively outside of the cathode; moreover, its "center" is set to be the center of mass of that volume (see Fig. 10). The thickness of the first layer is equal to the distance that a single test macroparticle would travel if subject to external forces only; this choice is justified by the fact that such a particle is screened from its image charge by the particles behind it. The particles to be simulated are than placed randomly inside the layer and given a longitudinal momentum which increases linearly with $z$ from the thermal momentum and the test particle momentum. The exit from the cathode of the subsequent layers occurs gradually in steps of fraction of picoseconds 

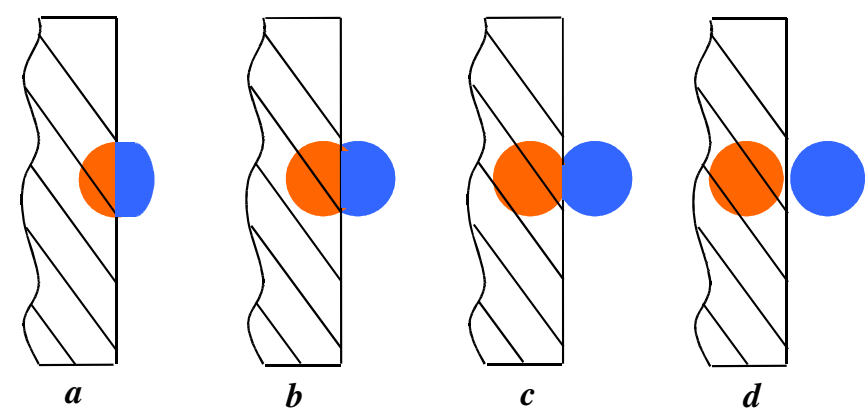

FIG. 10. (Color) Schematic sequence of the exit of the macroparticle from the cathode; in blue the particles, in red the images.

and is retarded by the presence of the images that moves symmetrically inside the cathode. Each particle is under the effect of the external fields, of the self-consistent e.m. forces evaluated taking into account the screening due to the distribution of the charge inside the macroparticle and under the force exerted by the images. When a layer of charges is completely out from the cathode, another layer is created and welded to the precedent in such a way that the longitudinal phase space of the beam does not present discontinuities; this is achieved in much the same manner as the first layer is produced except for the test particle, which is substituted by the closest particle of the previous layer directly "in front" of the new particle considered: this means the new test particle is extracted from a small transverse area ahead of the particle coming out. The process is repeated until the whole bunch has been extracted. The bunch length down the beam line turns out to be in agreement with other codes [12]. In Fig. 10 a sketch of the macroparticle extraction is shown. In Fig. 11 the actual extraction phase of the bunch is presented at four

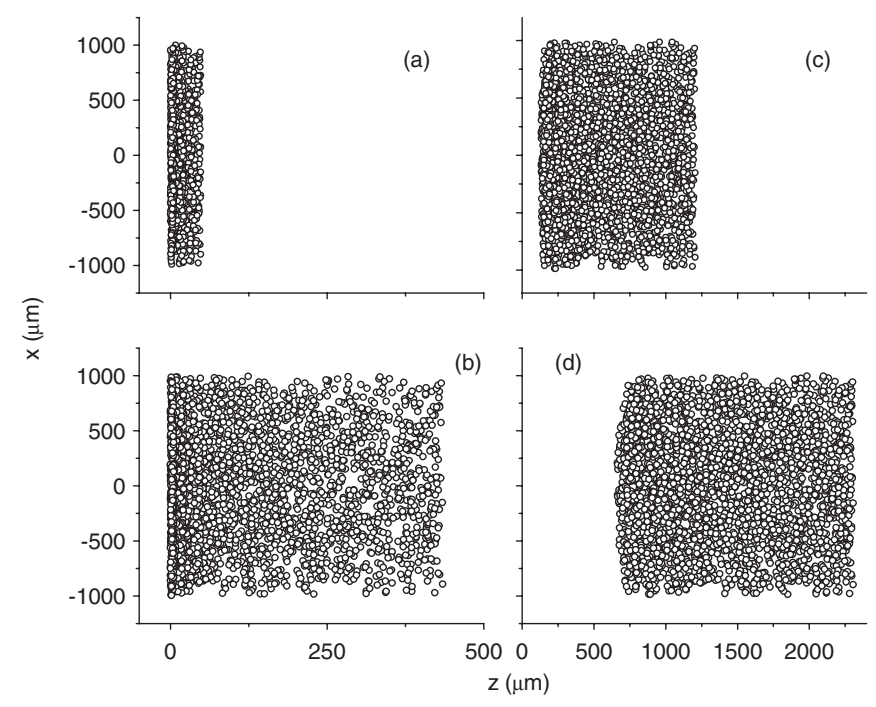

FIG. 11. A $(z, x)$ projection of a sample of the 20000 macroparticles of an $1 \mathrm{nC}$ electron bunch coming out from the photocathode: (a) $t=5 \mathrm{psec}$, (b) $t=10 \mathrm{psec}$, (c) $t=15 \mathrm{psec}$, and (d) $t=20$ psec.

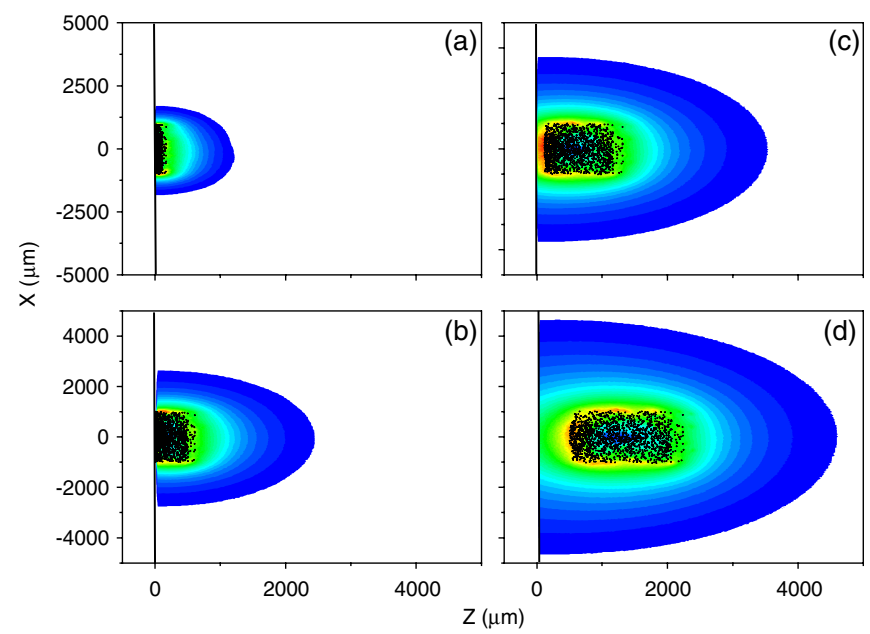

FIG. 12. (Color) $|\mathbf{E}|$ in the plane $(z, x)$ for (a) $t=5 \mathrm{psec}$, (b) $t=10 \mathrm{psec}$, (c) $t=15 \mathrm{psec}$, and (d) $t=20 \mathrm{psec}$. The black scatters are a sample of the 20000 macroparticles used in the simulation. Total charge is $1 \mathrm{nC}$

successive time instants. The contour plots of the modulus of the self-consistent electric field are presented in Fig. 12. As can be seen, the electric field is stronger on the trailing edge of the bunch during the extraction phase, where the bunch is denser. At the first instants of extraction a certain granularity is present on the structure of the field due to the discrete nature of the macroparticle scheme. A detailed simulation of bunch extraction and acceleration in the first stages of a linac, together with an estimation of the fields and of the power emitted, have been presented in $[10,11]$.

\section{B. Production of $\mathrm{THz}$ radiation}

The last example of an application of RETAR is the production of coherent terahertz radiation from an electron bunch passing through a bending magnet. Such radiation sources have received much interest recently, as for instance in the general and exhaustive paper by Geloni et al. [13], and THz sources are reported from different physical principles, such as transition radiation and edge radiation [14]. In the following we will focus on coherent synchrotron radiation, produced by a bending magnet identical to the one in Sec. IVD and the radiation is collected on a small $(\theta \approx 1 / \gamma)$ portion of a spherical surface with radius of $3 \mathrm{~m}$, centered in the midpoint of the magnet itself; the bunch has 25000 macroparticles. Since we are not interested in microbunching formation, particle interactions are switched off (i.e. only the external magnetic field is taken into account) and the self-consistent fields are evaluated only on the target screen.

The main differences with respect to the radiation produced by a single particle consist in the appearance of a dependence, in the radiation spectrum, on the bunch form factor [15]. In order to produce radiation in the range 0.1$1 \mathrm{THz}$, the bunch current profile we use is Gaussian with 


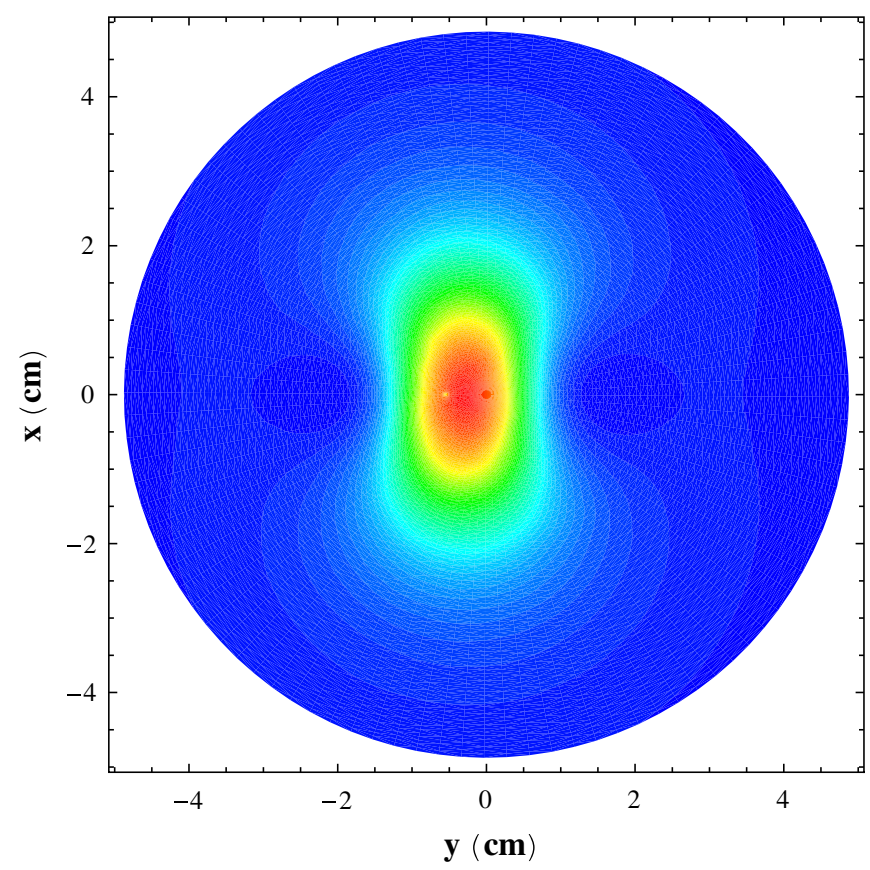

FIG. 13. (Color) Collected energy density for a Gaussian bunch.

$\sigma_{x}=\sigma_{y}=\sigma_{z}=100 \mu \mathrm{m}$, a charge of $300 \mathrm{pC}$, and an energy of $80 \mathrm{MeV}$. We performed two different simulations with different energy spread and emittance of the beam. For both situations the energy density collected on the screen (Fig. 13) is similar, much like the same result from the single particle setup, though the transverse span of the radiation and the energy collected are larger and depend on the beam transverse size. As stated before, we expect the spectrum to heavily depend on the form factor. In Fig. 14 we plot both the field Fourier transform and the form factor and the correspondence is very satisfactory when the bunch has no uncorrelated energy spread and emittance. Moreover, if both an energy spread $\delta E / E=$

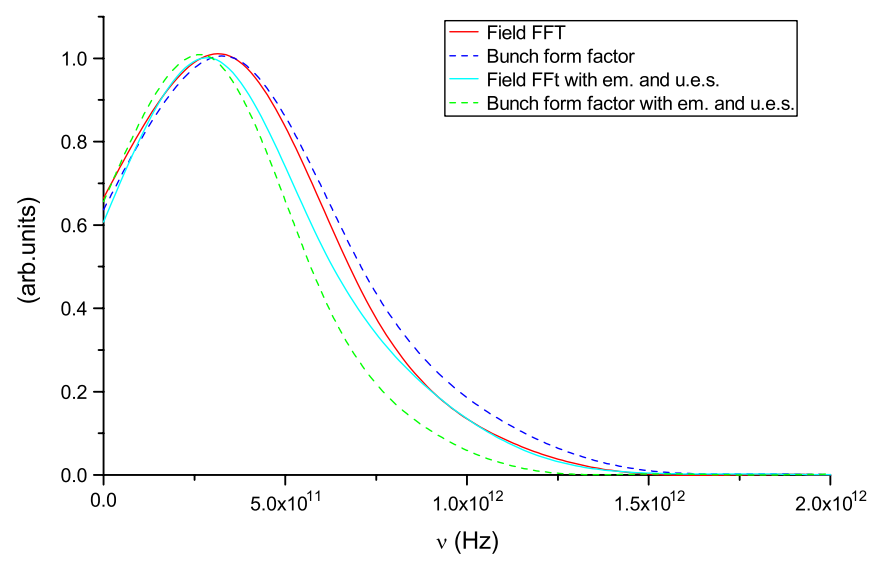

FIG. 14. (Color) Field fast Fourier transform (FFT) and bunch form factor for a beam with and without emittance and uncorrelated energy spread and for a bunch with $\delta E / E=0.6 \%$ and $\epsilon_{x}=\epsilon_{y}=3.5 \mu \mathrm{m}$.
$0.6 \%$ and an emittance $\epsilon_{x}=\epsilon_{y}=3.5 \mu \mathrm{m}$ are added to the beam, we notice that the peak frequency is slightly shifted towards lower frequencies and the spectrum width is larger. The former result can be ascribed to the emittance, since we kept constant the mean total energy, so an increase in transverse momentum implies a slight decrease in the longitudinal one, thus reducing the average synchrotron frequency. The widening of the spectrum, instead, is mostly due to energy spread that implies a wider range of synchrotron frequency within the bunch particles.

\section{CONCLUSIONS}

The numerical code RETAR for the evaluation of the radiation from bunches of charged particles in external and self-consistent fields has been introduced. The basic physics underlying the code, the numerical structure, and the benchmarking process have been presented. As a physical application, we have shown a few data regarding the production of terahertz radiation taking advantage of the passage of a high brightness electron beam through a bending magnet.

\section{ACKNOWLEDGMENTS}

The authors wish to thank M. Ferrario, L. Giannessi, M. Quattromini, C. Ronsivalle, and C. Vaccarezza for their help and useful suggestions in writing this paper.

[1] J.D. Jackson, Classical Electrodynamics (John Wiley \& Sons, New York, 1998), 3rd ed.

[2] M. Ferrario et al., in Proceedings of the 2nd ICFA Advanced Accelerators Workshop on the Physics of High Brightness Beams, UCLA Faculty Center, Los Angeles, CA, 1999 (World Scientific, Singapore, 2000), http:// www.slac.stanford.edu/pubs/slacpubs/8000/

[3] L. M. Young, Los Alamos National Laboratory Report No. LA-VR-96-1835, 2003.

[4] M. Borland, Argonne National Laboratory Advanced Photon Source Report No. LS-287, 2000.

[5] K. Floetmann, ASTRA, http://www.desy.de/mpyflo/Astra \_dokumentation/.

[6] L. Giannessi and M. Quattromini, Phys. Rev. ST Accel. Beams 6, 120101 (2003).

[7] F. Ciocci, L. Giannessi, A. Marranca, L. Mezi, and M. Quattromini, Nucl. Instrum. Methods Phys. Res., Sect. A 393, 434 (1997).

[8] M. Dohlus and T. Limberg, in Proceedings of the 26th International Free-Electron Laser Conference, Trieste, Italy, 2004 (Comitato Conferenze Elettra, Trieste, Italy, 2005).

[9] Y. Ding et al., in Proceedings of the 2007 Particle Accelerator Conference, Albuquerque, New Mexico, 2007 (IEEE, Albuquerque, New Mexico, 2007).

[10] A. Bacci et al., in Proceedings of the 2007 Particle Accelerator Conference, Albuquerque, New Mexico, 2007 (Ref. [9]). 
[11] A. Bacci et al., in Proceedings of the 10th European Particle Accelerator Conference, Edinburgh, Scotland, 2006 (EPS-AG, Edinburgh, Scotland, 2006).

[12] See http://pcfasci.fisica.unimi.it/Pagine/RETAR/RETAR. htm.

[13] G. Geloni et al., Opt. Commun. 276, 167 (2007).
[14] J. G. Neumann et al., J. Appl. Phys. 105, 053304 (2009); G. Andonian et al., Phys. Rev. ST Accel. Beams 12, 030701 (2009).

[15] S. Nodvick and D. S. Saxon, Phys. Rev. 96, 180 (1954); C. J. Hirschmugl, M. Sagurton, and G. P. Williams, Phys. Rev. A 44, 1316 (1991). 